Ann. Zootech., I976, 25 (2), I8I-I88.

\title{
INFLUENCE DU MODE \\ DE PRÉSENTATION D'UN ALIMENT COMPLET SUR LA VITESSE DU TRANSIT DIGESTIF ET LA DIGESTIBILITÉ CHEZ LE PONEY
}

\author{
R. WOLTER, Andrée IDURIX et J. C. LETOURNEAU \\ avec la collaboration technique de Jacqueline Laxdread, Martine Exius et A. Brexi \\ Laboratoire de Nutrition et d'Alimentation, I. N. R. A., \\ École nationale vétérinaire, \\ 6933 ' Lyon Cedex 1
}

RÉSUMÉ,

Un aliment complet est présenté sous trois formes différentes : farine, granulé, expansé. Il est distribué à cinq poneys placés en cage à métabolisme pour étudier les répercussions des traitements mécaniques et hydrothermiques sur la vitesse de transit et la digestibilité des rations.

Les vitesses moyennes du transit digestif ne sont pas influencées significativement par le type de présentation de l'aliment.

Les digestibilités de la matière sèche et de la matière organique, et surtout de l'extractif non azoté non amylacé, sont améliorées par la granulation et davantage par l'expansion, alors que l'utilisation digestive des matières azotées est légèrement diminuée. Ces traitements sont sans action sur la glycémie.

\section{INTRODUCTION}

Le conditionnement de la ration des équidés peut présenter de multiples arantages : facilités d'approvisionnement, de stockage et de distribution, réduction du gaspillage et suppression de la consommation sélective, contrôle précis et constant du niveau et de l'équilibre alimentaires, amélioration de la valeur nutritive et même de la valeur hygiénique des aliments.

- Avec des fourrages utilisés comme régime exclusif, HaEnLEIN, Hor.dren et YooN (I966) ont montré que la condensation (broyage, puis granulation) augmente nettement la consommation volontaire. Nos récents essais, conduits avec un foin 
de graminées distribué en quantités restreintes, ont mis en évidence que le broyage, suivi ou non de granulation, accélère le transit digestif (WOLTER, DURIX et LETour$\mathrm{NEAU}$, I974) et abaisse légèrement la digestibilité de la cellulose, sans affecter celle des autres constituants alimentaires (WOLTER, DURIX et LETOURNEAU, I975).

- Avec des rations à base de fourrages et de céréales, la condensation augmente l'ingestion volontaire, mais amoindrit quelque peu la digestibilité de la cellulose (HrNTz et Lox, rg66). Par ailleurs, les traitements thermiques qui interviennent dans la granulation et, à plus forte raison, ceux qui sont mis en œuvre dans les techniques de floconnage ou d'expansion, doivent accroître la valeur énergétique des aliments complets. Fin effet, ils favorisent 1'attaque enzymatique de l'extractif non azoté dans l'intestin grêle, conduisant à une résorption de glucose dont 1'efficacité énergétique est excellente. De ce fait même, ils minimisent les fermentations microbiennes du gros intestin, qui libèrent des acides gras volatils dont la production et l'utilisation métabolique s'accompagnent de pertes énergétiques bien supérieures.

A ce propos, nous comparons dans le présent essai trois types de présentation d'un même aliment complet, soit en farine, soit en granulés habituels, soit en granulés expansés. L'essai a été mis en place sur des poneys qui constituent de bons modèles pour l'étude des processus digestifs chez le Cheval (SLADE et HInTz, I969). Nos contrôles ont porté dans un premier temps sur le niveau d'ingestion en libre service, puis, alors que la consommation est restreinte, sur la vitesse du transit digestif et la digestibilité de l'aliment, ainsi que sur la glycémie.

\section{I. - CONDITIONS EXPÉRIMENTALES}

Six poneys mâles dont le poids moyen varie de $188 \mathrm{~kg}$ à $206 \mathrm{~kg}$ du début à la fin de l'expérimentation, sont placés en cages à métabolisme. L'un d'eux, le numéro 4 , tombé malade avant que soient terminées les mesures, a dû être retiré de l'essai. Les résultats rapportés sont donc les moyennes des valeurs obtenues à partir des cinq poneys sains.

Les animaux sont répartis en trois lots, et reçoivent alternativement, au cours de trois périodes successives de six semaines, un même aliment complet présenté soit en farine, soit en granulés, soit en expansé.

L'analyse granulométrique de la farine montre que la taille des particules se répartit de la manière suivante : supérieure à $0,4 \mathrm{~mm}$ pour $32 \mathrm{p}$. Ioo, comprise entre 0,3 et $0,4 \mathrm{~mm}$ pour $\mathrm{I}_{7}, 6$ p. Ioo, de 0,15 à $0,3 \mathrm{~mm}$ pour $13,8 \mathrm{p}$. I 00 , et inférieure à $0,15 \mathrm{~mm}$ pour $28 \mathrm{p}$. Ioo.

Un tiers de cette farinc est distribué tel quel, après humidification légère, au moment du repas; un autre tiers est mis en granulés de $4,5 \mathrm{~mm}$ de diamètre (presse EIB-CPH Europe) ; le troisième est expansé. Dans ce cas, la fabrication se fait au moyen d'un "expandeur "qui soumet la farine à une injection de vapeur ct à une très forte pression mécanique élevant la température de l'aliment vers $90^{\circ} \mathrm{C}$, et assurant une gélatinisation de l'amidon; la sortie à travers une filière est accompagnée d'une décompression brutale qui entraîne une forte augmentation du volume du granulé, justifiant le terme "expansé ".

La composition bromatologique et les résultats des analyses de ces aliments sont rassemblés dans le tableau $\mathbf{I}$.

Pendant les deux premières semaines de chaque période, les animaux disposent librement de l'aliment, de manière à mesurer les niveaux de consommation volontaire ; à partir de la troisième semaine, ils sont restreints à $4 \mathrm{~kg}(2,56 \mathrm{UF})$ par jour, et la ration est distribuée en deux fois, à $8 \mathrm{~h}$ et $\mathrm{r} 7 \mathrm{~h}$. Les récoltes de fèces sont effectuées pendant la quatrième semaine. Les trois périodes d'expérimentation se déroulent de la même manière et sont séparées entre elles par une semaine supplémentaire d'adaptation.

Les vitesses de transit ont été évaluées par la méthode des particules colorées déjà décrite (Wolter, Durix et Letourneau, I974). L'analyse des différents éléments : matières protéiques brutes (N Kjeldahl $\times 6,25$ ), cellulose brute (méthode de Scharrer), amidon (hydrolyse enzyma- 


\section{TABLEAU I}

Composition des aliments (en p. Ioo)

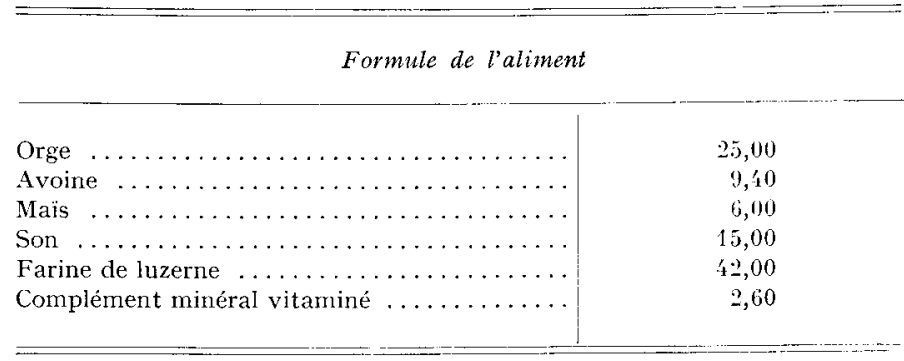

Résultats de l'aualyse chimique

\begin{tabular}{|c|c|c|c|}
\hline & I arine & Granulé & Expansé \\
\hline Matière sèche & 90,50 & $90,8: 2$ & 91,19 \\
\hline Protéines brutes $(\mathrm{N} \times(6,25) \ldots$ & 14,76 & 14,92 & 11,68 \\
\hline Cellulose (de Scharrer)........ & 16,12 & 15,35 & 11,68 \\
\hline Amidon $\ldots \ldots \ldots \ldots \ldots \ldots$ & 14,66 & 15,80 & 17,41 \\
\hline Minéraux .............. & 8,35 & 8,66 & 8,96 \\
\hline Calcium $\ldots \ldots \ldots \ldots \ldots \ldots$ & 1,49 & 1,65 & 1,61 \\
\hline Phosphore............. & 0,57 & 0,58 & 0,57 \\
\hline
\end{tabular}

tique: Charlier et al., I974), matières minérales totales (calcination à $55^{\circ} \mathrm{C}$ ), calcium (photométrie de flamme), phosphore (colorimétrie), a permis de calculer les coefficients d'utilisation digestive.

Les glycémies ont été déterminées à partir d'échantillons de sang jugulaire recueilli sur fluorure de sodium, avant la distribution de la ration, puis une heure trente et sept heures trente après celle-ci. Elles sont mesurées par la méthode colorimétrique de Folin et Wu (adaptée au photocolorimètre Lumétron, établissement Photovolt Corporation).

\section{II. - RÉSULTATS}

\section{I. - Consommation volontaire}

Lorsque l'aliment est laissé en libre disposition, au cours de la phase préliminaire de chaque période, les ingestions spontanées sont en moyenne les suivantes:

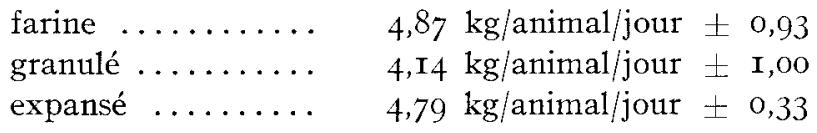

Elles ne sont pas significativement différentes. 


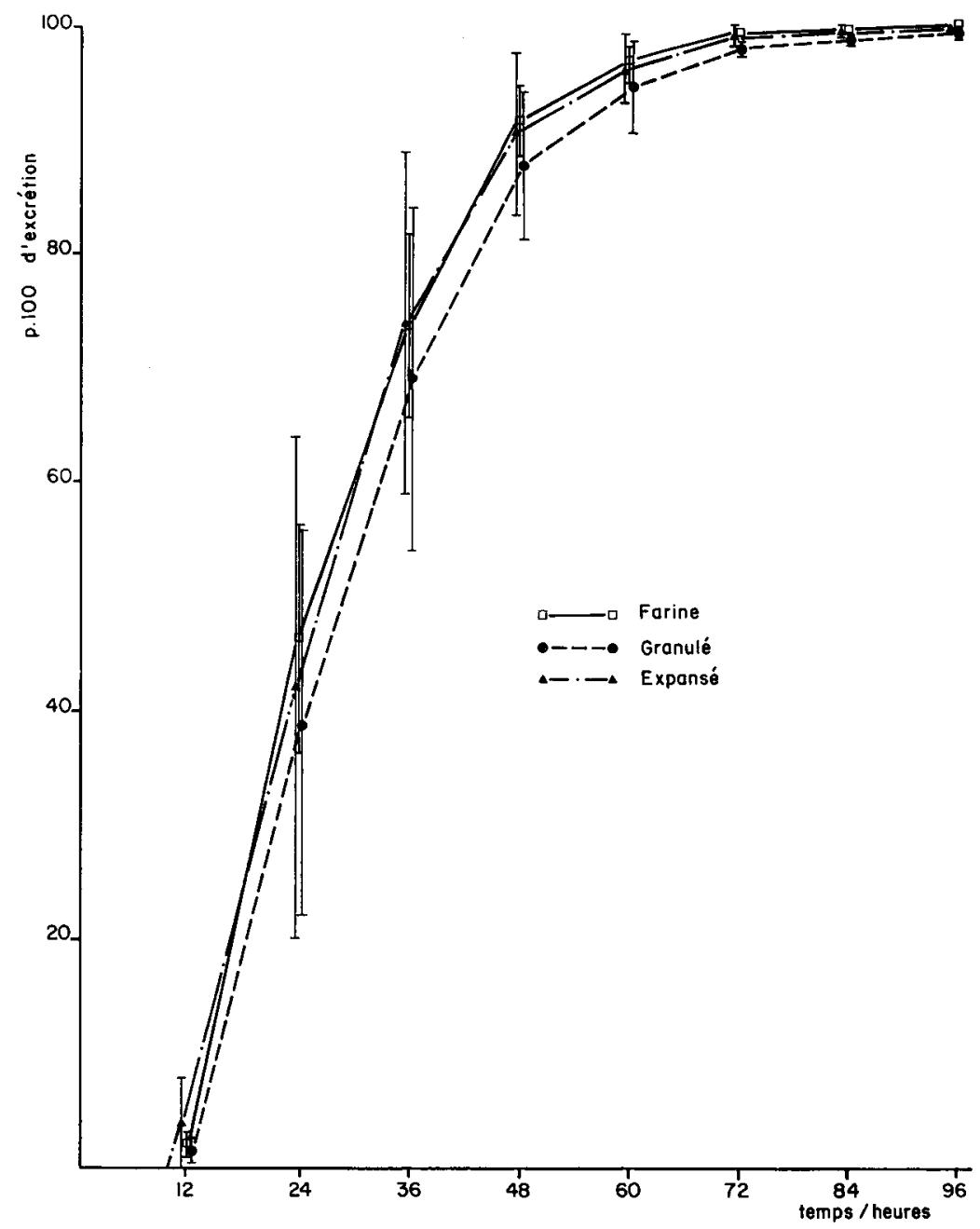

FIG. I. - Courbe d'élimination fécale des particules colorées (moyenne sur cinq poneys)

TABLEAU 2

Temps moyen de rétention digestive

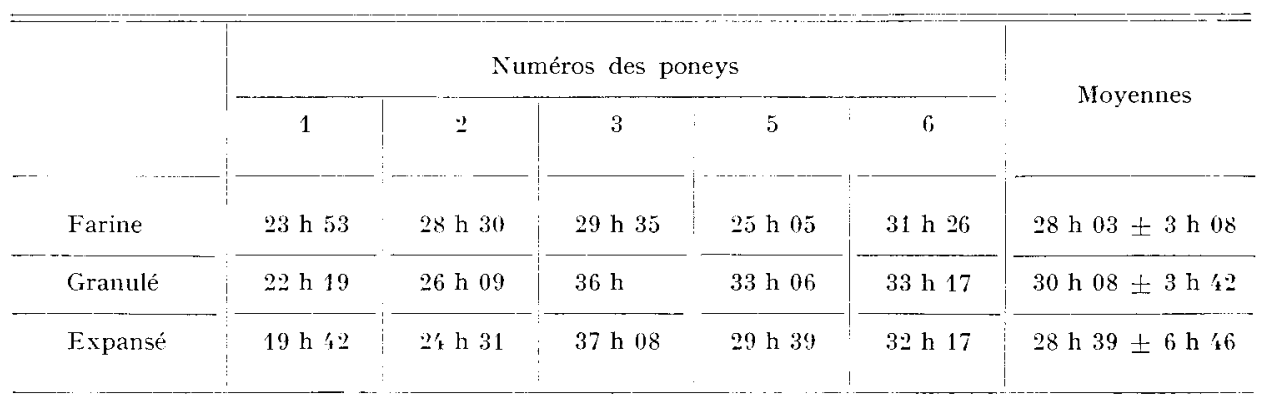

Les différences ne sont pas significatives. 


\section{2. - Vitesse $d u$ transit digestif (en alimentation restreinte)}

Les pourcentages cumulés de particules colorées retrouvées dans les matières fécales dans des intervalles de $\mathbf{I} 2$ heures, chez chacun des 5 poneys, permettent de construire, pour chaque présentation de l'aliment, les courbes moyennes d'élimination représentées sur la figure I.

Les courbes individuelles construites selon la méthode de CASTLE (I956) sont utilisées pour calculer les temps moyens de rétention digestive en fonction de la présentation physique de l'aliment complet (tabl. 2).

\section{3. - Digestibilité (en alimentation restreinte)}

Les coefficients moyens d'utilisation digestive, établis sur deux périodes expérimentales, ainsi que les taux de glucose plasmatique, sont réunis dans le tableau 3 .

TABLEAU 3

Coefficients d'utilisation digestive et taux plasmatique de glucose

(moyennes sur cinq poneys)

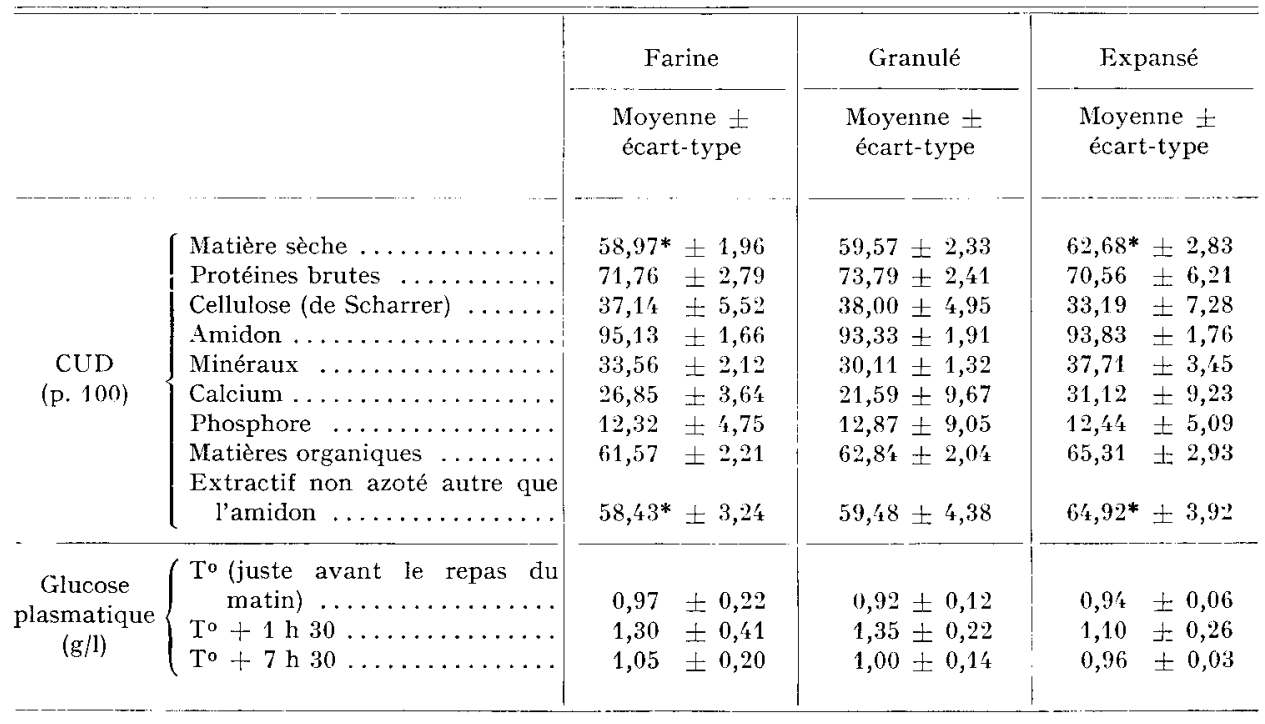

* Les résultats affectés d'un astérisque diffèrent significativement $(P<0,05)$.

\section{III. - DISCUSSION}

\section{I. - Lors de la libre distribution}

I a consommation est peu modifiée par le mode de traitement de l'aliment. Les quantités volontairement ingérées sont légèrement supérieures avec la farine et avec l'expansé (respectivement 4,87 et $4,79 \mathrm{~kg} /$ animal/jour) par rapport aux 
granulés (4,I4 kg/animal/jour). Ces valeurs ne sont toutefois pas significativement différentes. Les quantités consommées semblent dépendre moins dı conditionnement que de la dimension des particules, celle-ci étant la même pour les trois formes de présentations.

\section{2. - En alimentation rationnée (4 $\mathrm{kg} /$ animal/jour)}

a) Les temps moyens de rétention digestive restent compris entre $28 \mathrm{~h}$ et $30 \mathrm{~h}$, quelle que soit la présentation physique de l'aliment. Ils sont comparables à ceux que nous avons précédemment obtenus avec un foin de graminées (à 33 p. Ioo de cellulose), mis en farine ( $25 \mathrm{~h}$ ) ou en granulés ( $3 \mathrm{I} \mathrm{h}$ ) (WOLTER, DURIX et Litrourneau, I974). De même avec une farine de luzerne condensée (à 2I p. Ioo de cellulose), nous les avons évalués à 29 h 30 (résultats non publiés).

Dans le cas d'une alimentation restreinte, il apparait donc que le temps de rétention digestive est surtout lié à la taille des particules alimentaires. D'ailleurs, il a déjà été montré, chez le Poney, que les marqueurs particulaires de la phase solide ont une rapidité d'évacuation inversement proportionnelle à la dimension des particules (ARGENzIo et al., I974). De même chez les ruminants en libre consommation, broyage et granulation diminuent le temps de séjour de la ration d'autant plus que le broyage est plus fin (JoURNE'T et HODEN, I973).

Le traitement hydrothermique influence peu la durée du transit digestif. Chez le Porc par comparaison, le floconnage du mais a pour effet de ralentir la vidange stomacale de la phase soluble et d'accéléter celle de la phase solide (BoRGIDA, I975), alors que la granulation d'une farine n'a que peu de répercussion sur la ritesse du transit total (EECKHOUT, I972).

b) Par contre, le conditionnement de la ration retentit de manière plus sensible sur la digestibilité de certains constituants alimentaires. Ainsi, l'amélioration de l'utilisation digestive est significative pour la matière sèche et elle est près de l'être pour les matières organiques lorsqu'on passe de la farine, aux granulés, puis à l'expansé (tabl. 3). De même, Aumaitre et Dumont (I975) ont constaté, chez le Porcelet, que le traitement hydrothermique des céréales augmente la digestibilité de la matière sèche, des matières organiques et de l'azote. Chez les ruminants, la fermentescibilité accrue des amidons gélatinisés est, dans une certaine limite, favorable à l'engraissement, mais elle exagère les risques d'acidose ruminale et peut conduire à des résultats opposés.

Les coefficients d'utilisation digestive des matières protéiques sont légèrement plus faibles avec la farine (7I,7 p. I00) et avec l'expansé (70,5 p. I0o), qu'arec les granulés $(73,8$ p. I00). La digestion enzymatique précoce de la fraction azotée rend celle-ci peu tributaire du type de présentation, mais se tessent vraisemblablement des conséquences de la réaction de Maillard résultant des traitements thermiques.

Les digestibilités apparentes de la cellulose brute ne présentent pas de différences significatives. Une corrélation nette se vérifie entre la digestion de la cellulose et la rapidité du transit digestif qui conditionne la durée de l'attaque microbienne dans le gros intestin.

Les valeurs toujours élevées des digestibilités de l'amidon $(95,13$ p. Ioo pour la farine, 93,33 p. Ioo pour le granulé et 93,83 p. Ioo pour l'expansé) ne permettent pas de juger du réel intérêt des divers traitements. Cependant, il a été vérifié que 
les préparations hydrothermiques (floconnage, expansion...) des céréales, qui provoquent une gélatinisation partielle de l'amidon, en accélèrent la digestion dans 1'intestin grêle du Porc (Aumaitre et Dumont, I975), comme ils en activent la fermentation dans le rumen des polygastriques (BERANGER, THIVEND et JARRIGE, I972).

Par contre, l'amélioration de la digestibilité de l'ensemble de la matière sèche s'explique bien par l'augmentation significative de la digestibilité de l'extractif non azoté non amylacé avec 1'aliment expansé $(64,92 \pm 3,92)$ par rapport au granulé $\left(59,4^{8} \pm 4,38\right)$ et à la farine $\left(5^{8,43} \pm 3,24\right)$. Il ressort donc que c'est cette fraction glucidique à base de substances pectiques et d'hémicelluloses qui tire alors le meilleur bénéfice du traitement hydrothermique quant à la digestibilité chez les équidés.

Cependant, les taux de glucose plasmatique, estimés à différentes heures par rapport aux repas, ne varient pas significativement. Même si l'expansion favorise une digestion précoce des glucides avec une résorption supérieure de glucose, la bonne régulation hormonale de la glycémie chez le Cheval (Hintz, Argexzio et Schryver, I97I) en masque vraisemblablement les effets au niveau du sang.

Les différents traitements mécaniques et hydrothermiques envisagés (broyage, granulation, expansion), qui ont peu d'influence sur le temps moyen de rétention digestive de la ration, agissent par contre sur son efficacité alimentaire; en particulier, les digestibilités de la matière sèche et de la matière organique, et plus précisément de l'extractif non azoté non amylacé, sont améliorées par l'expansion. Cependant, ces résultats ne donnent qu'une appréciation globale des processus digestifs.

Nos études actuelles sur des poneys porteurs de fistules cacales permettront de préciser le site de digestion et la nature des nutriments absorbés, qui ont des conséquences notables sur l'hygiène digestive et l'efficacité de la ration.

Recu pour publication on octobre 1975.

\section{SUMMARY}

EFFECT OF THE PHYSICAI, HORM OF A COMPLETE MIXED FEED ON ITS RATE OF PASSAGE THROUGH THE DIGESTIVE TRACT AND DIGESTIBILITY IN PONEYS

A complete mixed feed was subjected to different treatments, either grinding, pelleting or popping, and offered to 5 poneys placed in metabolism crates. The aim of the experiment was to study the repercussions of mechanical or moist-heat treatments of the feed on its transit rate and cligestibility. the feed.

The average digestive transit rates were not significantly affected by the physical form of

The digestibilitics of dry matter and organic matter and especially that of the N-free extract without starch, were improved by pelleting and especially by popping, whereas the apparent digestibility of crude protein was slightly reduced. These treatments did not affect the blood glucose level. 


\section{RÉFÉRENCES BIBI,IOGRAPHIQUES}

Argenzio R. A., Lowe J. E., Piekard D. W., Stevins C. E., I974. Digesta passage ind water exchange in the equine large intestine. Am. J. Physiol, 226, 1035-1042.

Aimaitre A., Dumont R., I975. Utilisation des céréales dans les régimes de sevrage précoce du Porcelet : comparaison de l'orge et du blé et efficacité de quelques traitements hydrothermiques. Journées de recherche porcine en France, I $5 \mathrm{I}-\mathrm{I} 60$, I. N. R. A., I. T. P. éd., l’aris.

Beranger C., Thivend P., Jarrige R., I972. Influence des traitements mécaniques et hydrothermiques sur la valeur nutritive du mais chez les jeunes bovins à l'engrais. Ann. Zootech., 21, r75-19o.

BORGIDA L. P., I975. Floconnage et expansion de l'orge et du mais : étude technologique, digestion du mais floconné chez le Porc en croissance. Journées de recherche porcine en France, 99-IO4, I. N. R. A., I. T. P. éd., Paris.

CASTLE E. J., I956. The rate of passage of foodstuffs through the alimentary tract of the goat. I. Studies on adult animals fed on hay and concentrates. Brit. J. Futr., 10, I5-25.

Charler C., Van Eenaeme C., Carnat B., Pondant A., Lambot O., Bienfait J. M., I974. Méthode de dosage semi-automatique de l'amidon et du glucose dans les aliments pour bétail. Ann. Med. Vet., 118, $28 \mathrm{I}-294$.

Fickrout I. W., I972. Vitesse de passage de granulés et de farine à travers le tube gastro-intestinal des porcs Landrace belge et Piétrain. Revue de l'Agriculture, 3, $42 \mathrm{I}-433$.

Haenlein G. I. W., Holdren R. D., Yoon Y. M., I966. Comparative response of horses and sheep to different physical forms of alfalfa hay. J. Anim. Sci., 25, 740-743.

Hintz H. F., Loy R. G., 1966. Effects of pelleting on the nutritive value of horse rations. I. Anim. Sici., 25, 1059-1062.

Hintz H. F., Argenzio R. A., Scirryer H. F., rg7r. Digestion coefficients, blood glucose levels and molar percentage of volatile acids in intestinal fluid ponies fed varying forage grain ratios. J. Anim. Sci., 33, 992-995.

Journet M., Hoden A., 1973. Utilisation des luzernes et des graminées déshydratées comme unique fourrage de la ration des vaches laitières et étude de leur digestion dans le rumen. Ann. Zootech., 22, 37-54.

Si.ADE L. M., H1xt2 H. I., I 969 . Comparison of digestion in horses, ponies, rabbits and guinea pigs. J. Anim. Sci., 28, 842-843.

Wolter R., Detrix Andrée, Letourniay J. C., I974. Influence du mode de présentation du fourrage sur la vitesse du transit digestif chea le Poney. Ann. Zootech., 3, 293-300.

Wolter R., Junix Andrée, Letourneau J. C., i975. Influence du mode de présentation du fourrage sur la digestibilité chez le Poney. Ann. Zootech., 24, 237-242. 